\title{
Desarrollo de membranas de quitosano y diseño de un equipo para la eliminación de metales pesados del agua
}

\author{
Chitosan membrane development and \\ design of equipment for the removal \\ of heavy metals from water
}

\author{
Jesús Mora Molina' \\ Luis Chaves Barquero² \\ Mario Araya Marchena ${ }^{3}$ \\ Ricardo Starbird Pérez ${ }^{4}$
}

Mora, J; Chaves, L; Araya, M; Starbird, R. Desarrollo de membranas de quitosano y diseño de un equipo para la eliminación de metales pesados del agua. Tecnología en Marcha. Vol. 25, No 3. Julio-Setiembre 2012. Pág 3-18.

2. Químico. Investigador del Centro de Investigación en Protección Ambiental (CIPA), Escuela de Química, Tecnológico de Costa Rica. Correo electrónico: luis.chavesb@gmail.com

3. Químico. Investigador del Centro de Investigación en Protección Ambiental (CIPA), Escuela de Química, Tecnológico de Costa Rica. Correo electrónico: marayam8I @gmail.com

4. Químico. Investigador del Centro de Investigación en Protección Ambiental (CIPA), Escuela de Química, Tecnológico de Costa Rica. Correo electrónico: ricardostarbird@gmail.com 


\section{Resumen}

El presente estudio comparó la eficiencia de la filtración con membranas de quitosano I,75\% m/v, entrecruzadas con glutaraldehído $(0,08 \% \mathrm{~m} / \mathrm{v})$ y $\sin$ entrecruzar, para estimar la capacidad de remoción de iones de cadmio, cromo y cobre de disoluciones modelo. Además, se diseñó un equipo de bajo costo para la experimentación con las membranas elaboradas.

La finalidad de la investigación era emplear materiales biodegradables para remover metales pesados de aguas, mediante una técnica de bajo consumo energético $y$, por otra parte, generar soluciones baratas, efectivas y aplicables a problemas específicos.

Se elaboraron dos fichas técnicas con información sobre las membranas y se encontró que el cromo fue el metal removido en mayor medida por las membranas entrecruzadas, ajustándose al modelo de isoterma de Freundlich.

Sin embargo, no se encontró relación entre el tamaño de poro de las membranas y el grado de entrecruzamiento.

\section{Palabras clave}

Membranas de quitosano, remoción por filtración, metales pesados, disoluciones modelo.

\section{Abstract}

A filtration technique with $1,75 \% \mathrm{~m} / \mathrm{v}$ chitosan membranes crosslinked with glutaraldehyde $(0,08 \%$ $\mathrm{v} / \mathrm{v}$ ) was used to quantify the removal capacity of chromium, copper and cadmium ions from water. A simple and low cost filtration system was developed to use with prepared membranes.

The main goal was to use biodegradable materials for removing heavy metals from water, through a low energy consumption, cheap, and specific method.

As a result, two data sheets were prepared for the membranes. It was found out that chromium was the metal with the highest removal from water, by using a crosslinked membrane. Metal adsorption was best adjusted to the Freundlich isotherm model, better than Langmuir isotherm model.

However, it was found no correlation between pore size and crosslinking degree.

\section{Key words}

Chitosan membranes, removal by filtration, heavy metals, model solutions. 


\section{Introducción}

La introducción repentina de sustancias químicas de procedencia industrial y agrícola puede abatir la capacidad de auto limpieza de los ecosistemas receptores y por lo tanto dar como resultado la acumulación de contaminantes a niveles problemáticos y hasta perjudiciales.' Muchas de las aguas de desecho, por ejemplo, podrían ser empleadas en tierras de cultivo para aprovechar su contenido de minerales y darle mejores propiedades físicas al suelo.

Sin embargo, la presencia de metales pesados en estas aguas y su acumulación en el suelo aumentarían la toxicidad en los cultivos y podrían afectar las fuentes de agua de consumo humano. ${ }^{2}$ El uso de tecnologías de bajo costo, como la filtración con membranas amigables con el medio ambiente, permitiría tratar este tipo de aguas contaminadas.

La membrana es un material capaz de separar sustancias en función de sus propiedades físicas y químicas mediante el empleo de una fuerza directora. ${ }^{3}$ La tecnología de membranas de filtración en el tratamiento de aguas residuales ha tenido un auge en los últimos años, gracias a que estas no requieren aditivos químicos y son efectivas para retener metales pesados.

No obstante, el costo de fabricación de estas membranas es alto, por lo que es importante el desarrollo de membranas con biomateriales que sean más baratos, amigables con el ambiente y de fácil elaboración de acuerdo con objetivos específicos.

Una sustancia de interés en el tratamiento de aguas contaminadas con metales pesados es el quitosano y la quitina, consideradas como sustancias "ambientalmente amigables". Entre los principales usos que se hacen en la actualidad de estos biomateriales, y algunos de sus derivados, tenemos los siguientes:

- Coagulante primario para aguas residuales de alta turbidez y alta alcalinidad.

- Floculante para la remoción de partículas coloidales sólidas y aceites de pescado.

- Captura de metales pesados y pesticidas en soluciones acuosas. Algunos copolímeros de injerto del quitosano muestran alta efectividad para remover metales pesados. ${ }^{4}$
Trimukhe y Varma (2008) emplearon el quitosano entrecruzado para la acomplejación de metales pesados con buenos resultados en la eliminación de hierro, cobre y plomo. ${ }^{5}$ Song et al. (2007) utilizaron celulosa con injertos de quitosano como resina de intercambio para remover metales pesados del agua, las cuales mostraron alta selectividad a bajas concentraciones. ${ }^{6}$

Mientras que Aroua et al. (2007) usaron el quitosano en solución para acomplejar metales con un $50 \%$ de remoción máxima.7 Sin embargo, Paulino et al. (2007) emplearon quitosano con diferentes grados de acetilación para absorber plomo y níquel, mostrando absorciones específicas (diferentes valores en los parámetros de las isotermas de Freundlich y Langmuir). ${ }^{8}$ El estado del arte del uso del quitosano en la remoción de metales pesados permite estimar que este puede ejercer interacciones con metales pesados de manera específica y presentando una gran gama de formulaciones.

La capacidad de interacción del quitosano con metales pesados es mencionada por Chui et al. (1996)9, aduciendo que los aminoazúcares de la quitina y el quitosano tienen sitios efectivos de enlace con iones metálicos, formando complejos estables de coordinación.

Los electrones del nitrógeno presentes en los grupos aminos y $\mathrm{N}$-acetilos pueden enlazarse con los orbitales de transición de los metales, algunos grupos hidroxilos en los biopolímeros pueden funcionar como donadores. Por ende, los grupos hidroxilos desprotonados pueden interaccionar en sistemas de coordinación con iones metálicos. ${ }^{9}$

El quitosano es una poli-2-amino-2-deoxi-(I,4)D-glucopiranosa, un derivado de la quitina poli-2acetamida-2-deoxi-( I,4)-D-glucopiranosa (figura I). La quitina es el segundo biopolímero más abundante en la naturaleza, obtenido primariamente como subproducto de las especies crustáceas marinas.

El quitosano y sus derivados han tenido un gran auge en sus aplicaciones, debido a actividades como antitumoral, antiulcérico, anticuagulante y antimicrobial, además de sus aplicaciones farmacológicas y médicas; esto se debe a sus propiedades biodegradables, de baja toxicidad y buena compatibilidad. ${ }^{10}$ 


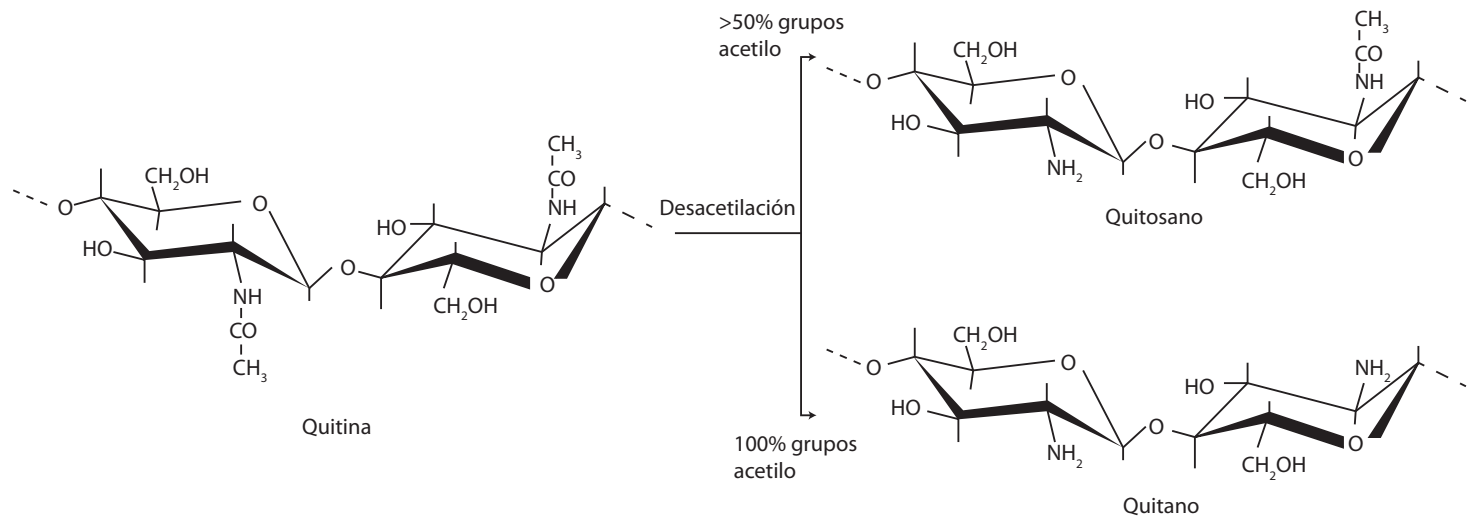

Figura I. Relación estructural entre la quitina, el quitosano y el quitano4.

Los efectos de los metales sobre el funcionamiento de los ecosistemas varían considerablemente y tienen importancia económica y en la salud pública. Los metales pesados actúan por mecanismos tales que afectan procesos biológicos como el desplazamiento de iones metálicos esenciales en las biomoléculas y el bloqueo de sus grupos funcionales, modificando la conformación activa de las biomoléculas.

En el caso del cadmio, este es un metal pesado contaminante de suelos, alimentos y ecosistemas. El cadmio es captado en ambientes y entra en la cadena alimentaria que puede afectar la salud humana. Hay suficiente evidencia de su posible acumulación tóxica y cancerígena durante exposiciones severas y crónicas. Walkley et al. (1988)" menciona que las concentraciones de 50-170 mg de Cd por kg de tierra están presentes en suelos australianos en forma de superfosfato (empleado como fertilizante).

Cook y Freney (1988)"' reportan un promedio de fertilizantes con fósforo de 459 mg de Cd por $\mathrm{kg}$ de fósforo en Australia." Esto implica que la agroindustria genera una importante entrada de este metal a las áreas de cultivo así como al ecosistema anexo.

Además, la especie cromo $\left(\mathrm{Cr}^{6+}\right)$ ha sido muy reportada debido a su toxicidad en ecosistemas y su necesidad de ser removidos de las aguas de desecho antes de su descarga a los sistemas de alcantarillado. La presencia de iones cobre $\left(\mathrm{Cu}^{2+}\right)$ en aguas de desecho también es un problema muy extendido en las industrias eléctrica, de cuero y de pinturas fungicidas. Cuando se ingiere en altas concentraciones, puede llegar a ser tóxico en humanos, causando también cáncer. ${ }^{9}$

Por lo tanto, la aplicación de una tecnología de bajo impacto energético, como la filtración con membranas de quitosano, para la remoción de metales pesados ( $\mathrm{Cd}, \mathrm{Cr}$ y $\mathrm{Cu}$ ) de las aguas residuales evitaría la contaminación de los mantos acuíferos, aguas de consumo humano, alimentos y la bioacumulación, con lo cual se disminuye el impacto en la salud y el ambiente, con costos reducidos de inversión y mantenimiento por medio de tecnologías limpias.

\section{Metodología}

\section{Materiales requeridos en la investigación}

Se utilizó quitosano de alto o medio peso molecular con grados de desacetilación mayores al 65\%. Se empleó acido acético glacial, solución de glutaraldehído, ácido nítrico, ácido clorhídrico, hidróxido de sodio, carbonato de sodio, con grado reactivo y cloruro de cobre $\left(\mathrm{CuCl}_{2}\right)$, dicromato de potasio $\left(\mathrm{K}_{2} \mathrm{Cr}_{2} \mathrm{O}_{7}\right)$ y cloruro de cadmio $\left(\mathrm{CdCl}_{2}\right)$, con grado analítico, además de patrones de grado Absorción Atómica de cadmio, cobre y cromo.

\section{Diseño experimental}

Las variables estudiadas fueron: porcentaje de quitosano utilizado en la preparación de las membranas, porcentaje de entrecruzante y cantidad de metales pesados en solución, dejando como constantes el grado de acetilación del quitosano 


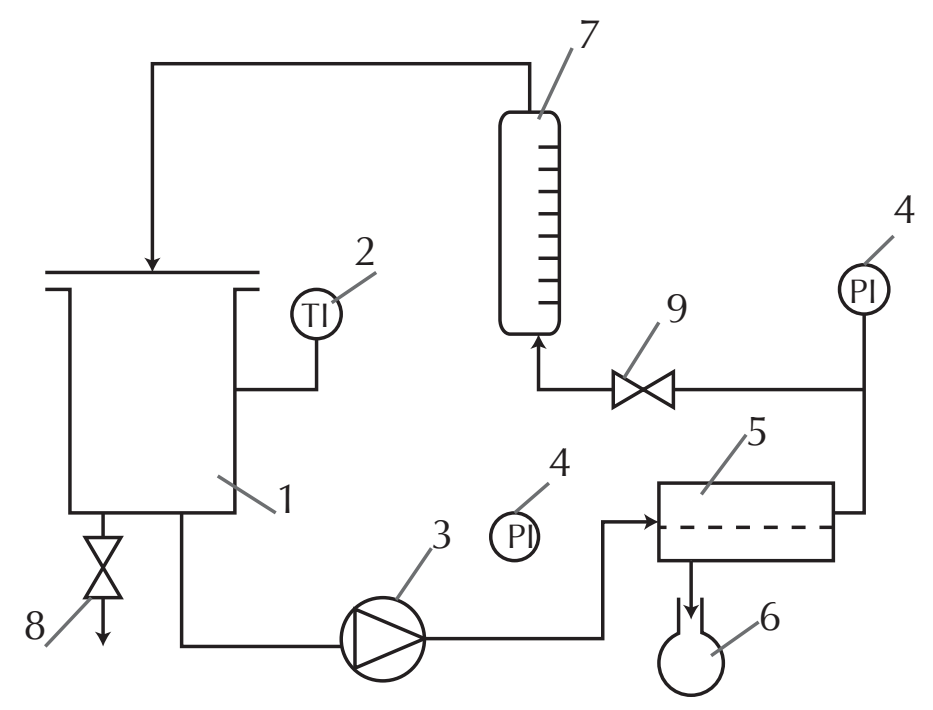

Figura 2. Equipo de laboratorio con membrana de quitosano: (I) tanque de alimentación, (2) termómetro, (3) bomba para membranas, (4) manómetro, (5) módulo de la membrana, (6) recolector del filtrado, (7) flujómetro, (8) válvula de salida, (9) válvula para regular la presión.

y el porcentaje de ácido acético empleado como solvente.

\section{Preparación de las membranas de quitosano}

La masa necesaria de quitosano fue disuelta en ácido acético al 1,5\% v/v a temperatura ambiente. Esta disolución fue extendida en cajas petri y sometida a una atmósfera de amoníaco por 12 horas. Luego, las membranas fueron lavadas con ácido acético al I,5\% v/v y agua desionizada y colocadas en una estufa a $45^{\circ} \mathrm{C}$ por ocho horas. Posteriormente, se dio el entrecruzamiento con glutaraldehído de varias concentraciones, exponiendo la película a la acción de $5 \mathrm{~mL}$ de la disolución entrecruzante por 2-5 minutos. Las membranas resultantes se secaron a $45^{\circ} \mathrm{C}$ en la estufa, hasta alcanzar la consistencia apropiada.

\section{Caracterización de las membranas}

Para la caracterización por espectroscopía infrarroja de las membranas de quitosano, se empleó un Nicolet 380 FT-IR del Centro de Investigación en Protección Ambiental del Tecnológico de Costa Rica.

Para determinar el tamaño de los poros de las membranas, se empleó microscopía de barrido electrónico (SEM).
Se utilizó un Microscopio Electrónico Jeol modelo JSM-6400F, de la Escuela de Materiales del Tecnológico de Costa Rica.

Se midieron las propiedades mecánicas de tensión por medio de un Tensilon de Shimadzu AG-I de la Unidad de Materiales del Instituto Nacional de Aprendizaje.

Cuantificación de los metales pesados en el agua

La determinación de las cantidades de metales pesados en solución previa y post filtración se realizó por medio de un equipo de Absorción atómica Perkin Elmer Aanalyst 800, en el Laboratorio de Servicios Químicos y Microbiológicos (Ceqiatec), siguiendo la metodología establecida en el "Standard Methods for the Examination of Waters and Wastewaters", vigésima edición. ${ }^{12}$

\section{Equipo de filtración}

Se diseñó un equipo según la figura 2, el cual se utilizó para la filtración de las disoluciones modelo de cromo, cadmio y cobre. Este equipo se diseñó lo más sencillo posible para minimizar costos, haciendo uso de materiales de fácil acceso, tales como manómetro, llave de paso, bomba de agua y multímetro. 


\section{Resultados y discusión}

Preparación de membranas de quitosano

La preparación de las membranas se realizó con las siguientes concentraciones de quitosano $(\% \mathrm{~m} / \mathrm{v})$ : $0,75 \%, 0,88 \%, \quad 1,0 \%, \quad 1,5 \%$ y $1,75 \%$. El disolvente utilizado fue una disolución acuosa I,5\% v/v de ácido acético. Estas membranas se prepararon mediante varios métodos: sin entrecruzar y entrecruzadas con glutaraldehído a las siguientes concentraciones (\%v/v): 0,31\%, 0, $16 \%$ y 0,08\%. Como coadyuvante de la flexibilidad, se agregó glicerina al $5 \% \mathrm{~m} / \mathrm{v}$.

Es importante señalar que, durante el proceso, muchas de las preparaciones no produjeron membranas en buen estado sino hidrogeles poco rígidos, que no permitieron completar el proceso de preparación. Esto fue parte de la optimización de las condiciones de trabajo con los recursos existentes, proceso que consumió varios meses de trabajo experimental, dado que se realizaron 17 combinaciones diferentes de las variables: concentración de quitosano y concentración de glutaraldehído.
A partir de las observaciones presentes en el cuadro I, se deduce que para todas las membranas sin entrecruzar se obtuvo un grado de flexibilidad relativamente alto. Sin embargo, para el proceso de filtración es muy importante el espesor de la membrana, ya que si es alto se espera una mayor área de contacto, pero si es bajo se espera un menor contrarreflujo. En los casos en los que la concentración de quitosano fue de $0,75 \% \mathrm{~m} / \mathrm{v}$, $0,88 \% \mathrm{~m} / \mathrm{v}$ y $1,0 \% \mathrm{~m} / \mathrm{v}$, el espesor de las membranas fue demasiado bajo para poder ser utilizadas en una filtración: las membranas se destruían al contacto con un flujo de agua de I litro por minuto, además, eran muy quebradizas debido al entrecruzamiento.

Al preparar las membranas con una concentración de I,5\% m/v en quitosano, se observó una mejora en el espesor obtenido.Además, las membranas ya no eran tan quebradizas como en el caso de concentraciones menores. Sin embargo, estas membranas aún no cumplieron con las características para poder ser utilizadas en el equipo de filtración, dado que eran quebradizas después del entrecruzamiento con glutaraldehído en las concentraciones empleadas.

Cuadro I. Características de las membranas preparadas.

\begin{tabular}{|c|c|c|c|}
\hline \multirow[b]{2}{*}{$\begin{array}{l}\text { Concentración de } \\
\text { quitosano }(\% \mathrm{~m} / \mathrm{v})\end{array}$} & \multirow{2}{*}{$\begin{array}{l}\text { Concentración de } \\
\text { glutaraldehído (\%v/v) }\end{array}$} & \multicolumn{2}{|c|}{ Observaciones sobre la membrana } \\
\hline & & Flexibilidad & $\begin{array}{l}\text { Resistencia al } \\
\text { hichamiento }\end{array}$ \\
\hline $0,75 \%$ & Sin entrecruzar & I & $\mathrm{A}$ \\
\hline $0,75 \%$ & 0,31 & 2 & A \\
\hline $0,75 \%$ & 0,16 & 2 & A \\
\hline $0,88 \%$ & Sin entrecruzar & 1 & A \\
\hline $0,88 \%$ & 0,31 & 2 & A \\
\hline $0,88 \%$ & 0,16 & 2 & A \\
\hline $1,0 \%$ & Sin entrecruzar & 1 & A \\
\hline $1,0 \%$ & 0,31 & 2 & A \\
\hline $1,0 \%$ & 0,16 & 2 & A \\
\hline $1,5 \%$ & Sin entrecruzar & I & B \\
\hline $1,5 \%$ & 0,31 & 2 & $\mathrm{~B}$ \\
\hline $1,5 \%$ & 0,16 & 2 & $\mathrm{~B}$ \\
\hline $1,5 \%$ & 0,08 & 2 & $\mathrm{~B}$ \\
\hline I,75\% & Sin entrecruzar & 1 & C \\
\hline I,75\% & 0,31 & 2 & C \\
\hline $1,75 \%$ & 0,16 & 2 & C \\
\hline I,75\% & 0,08 & I & C \\
\hline
\end{tabular}

Nota: | = membrana flexible, 2 = membrana quebradiza, $A$ = poca o nula resistencia, $B=$ resistencia limitada, $\mathrm{C}=$ mejor resistencia. 
(a)

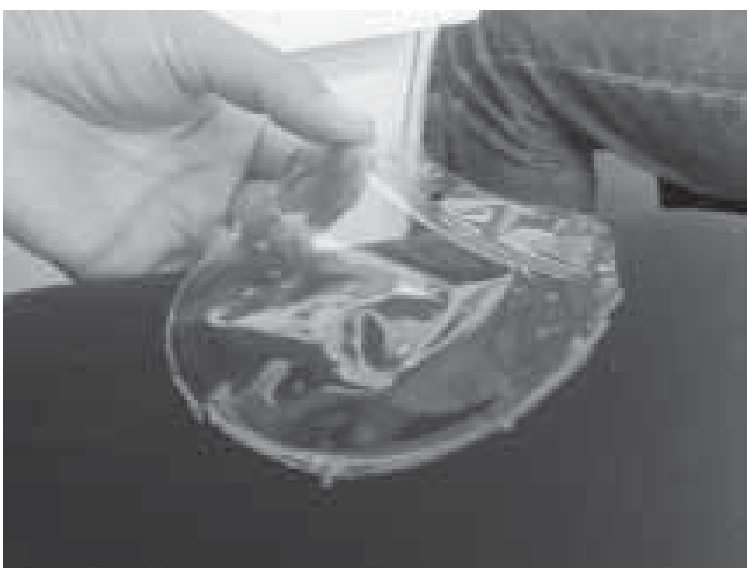

(b)

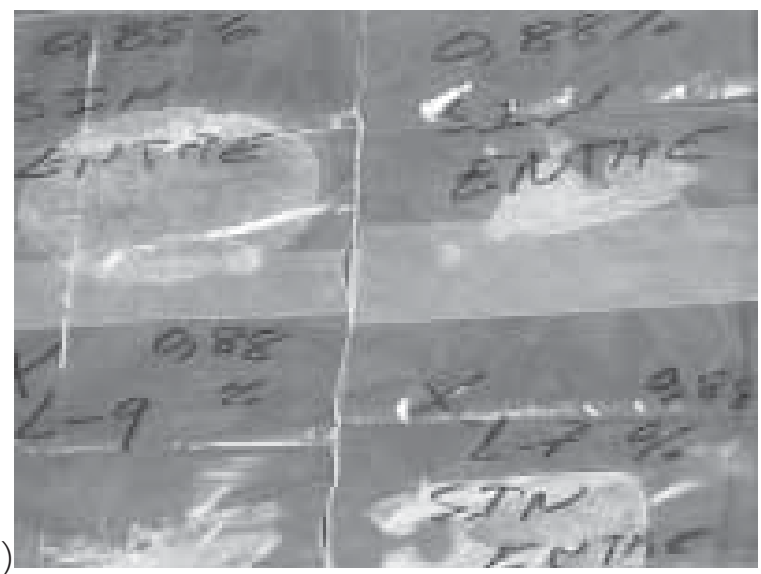

Figura 3. (a) Membrana de quitosanol,75\%m/v entrecruzada con glutaraldehído $0,08 \% \mathrm{v} / \mathrm{v}$. (b) membranas de quitosano no aptas para la filtración.

Las membranas que reunieron las mejores características, tanto en espesor como en resistencia al proceso de hinchamiento, fueron las preparadas a una concentración de $1,75 \% \mathrm{~m} / \mathrm{v}$ en quitosano. Se observó que al entrecruzar con glutaraldehído al 0,08\% v/v, las propiedades de flexibilidad de las membranas mejoraron significativamente. Por esta razón, se eligió esta membrana para llevar a cabo el proceso de filtración.

\section{Elaboración de fichas técnicas de los dos tipos de membranas empleados}

Se elaboró una ficha técnica por cada membrana preparada. Esta ficha incluye información estructural sobre las propiedades mecánicas de las membranas, así como su espectro infrarrojo y una imagen de microscopía electrónica SEM.

Los espectros de infrarrojo obtenidos para las membranas de quitosano, entrecruzadas y sin entrecruzar, permitieron una caracterización cualitativa de la estructura química presente en las membranas. Las bandas presentes en la zona de $3400 \mathrm{~cm}^{-1}$ aproximadamente corresponden a las vibraciones de los múltiples grupos hidroxilo de la estructura, así como los grupos amino $\left(-\mathrm{NH}_{2}\right)$ presentes.

A $1600 \mathrm{~cm}^{-1}$ aproximadamente, se observan bandas para grupos carbonilo, cerca de $1000 \mathrm{~cm}^{-1}$ se pueden observar las bandas para los enlaces C-O que funcionan como puente de unión de los monómeros que forman el quitosano (figura 4). En dichos espectros, se puede observar que el entrecruzamiento con glutaraldehído no provocó cambios drásticos en la configuración de las bandas en los espectros realizados.

Esto se debe a que el glutaraldehído se utilizó en concentraciones bajas con el principal objetivo de mejorar propiedades de elasticidad y resistencia de las membranas. Dichas modificaciones no alteran en gran medida las bandas que se muestran en el espectro, salvo la intensidad de la banda a $1600 \mathrm{~cm}^{-1}$, la cual es mayor en las membranas entrecruzadas, debido a la formación de un doble enlace $\mathrm{C}=\mathrm{N}$.

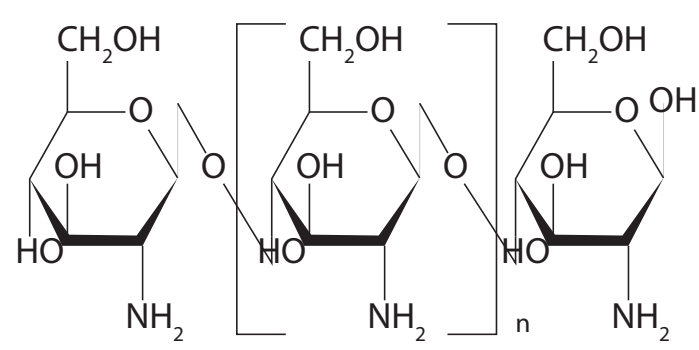

Chitosan

Figura 4. Estructura química del quitosano.

El proceso de entrecruzamiento entre el quitosano y el glutaraldehído tiende a disminuir las propiedades elásticas de las membranas de quitosano (20,7\%) en comparación con las membranas entrecruzadas (10,7\%). Sin embargo, se incrementa el módulo en las membranas entrecruzadas en más de tres veces con respecto a las membrana sin entrecruzar. 


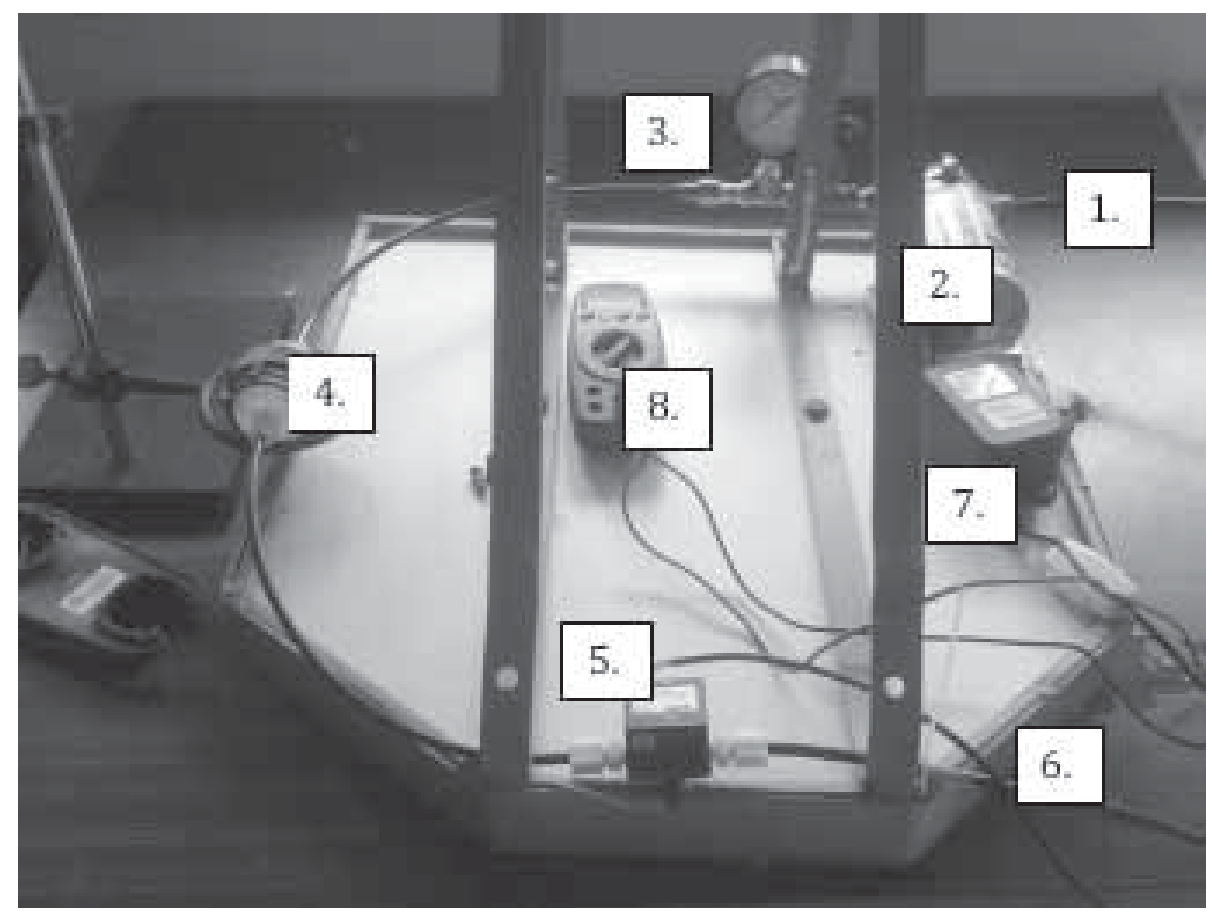

Figura 5. Equipo de laboratorio con membrana de quitosano: (I) manguera de alimentación del agua residual, (2) bomba para membranas, (3) manómetro, (4) módulo de la membrana, (5) medidor de flujo, (6) manguera salida del filtrado, (7) transformador de corriente, (8) medidor de voltaje.

Esto coincide con lo reportado para procesos de entrecruzamiento donde se busca incrementar la resistencia de la membrana (incremento del módulo elástico), rigidez y tenacidad (disminución del porcentaje de elongación).13

El proceso de entrecruzamiento busca mejorar las propiedades mecánicas de las membranas, además de variar la relación superficie-área para maximizar la capacidad de adsorción y modificar además las limitaciones hidrodinámicas como son la obstrucción de la columna y la pérdida de fricción. Durante los experimentos realizados, logró comprobarse que un exceso de glutaraldehído aumentaba la rigidez en tal medida que la membrana se volvía quebradiza. La cantidad utilizada finalmente permitió obtener membranas razonablemente flexibles y con capacidad de adsorción de metales.

\section{Diseño del equipo}

Se diseñó un sistema de filtración a escala de laboratorio para ser empleado con las membranas de quitosano preparadas. Este equipo se muestra en la figura 5. Consta de una bomba eléctrica de alimentación, la cual transfiere el agua desde un contenedor principal hacia una llave de paso acoplada a un manómetro, en el cual se mide la presión. Posteriormente, el agua pasa a través del módulo de la membrana, en el cual se encuentra la membrana en posición vertical para realizar la filtración. Luego de la filtración, el agua atraviesa un medidor eléctrico de flujo, acoplado a un medidor de voltaje. Este sistema permitió trabajar con un flujo de un litro por minuto, aproximadamente.

\section{Isotermas de Freundlich y Langmuir}

La filtración de las disoluciones modelo de metales se realizó en el equipo de filtración diseñado por el grupo de investigación. Se filtró un volumen de $250 \mathrm{~mL}$, a una velocidad de flujo de I litro por minuto. Para cada disolución preparada se realizó una filtración en una membrana de $1,75 \% \mathrm{~m} / \mathrm{v}$, tanto entrecruzada con glutaraldehído 0,08\% v/v como sin entrecruzar.

Para el estudio del proceso de adsorción de metales se prepararon varias disoluciones modelo a partir de patrones primarios sólidos, o patrones líquidos certificados. Estas disoluciones fueron las que se emplearon para la filtración. 
Cuadro 2. Ficha técnica de la membrana de quitosano al I,75\% $\mathrm{m} / \mathrm{v}$.

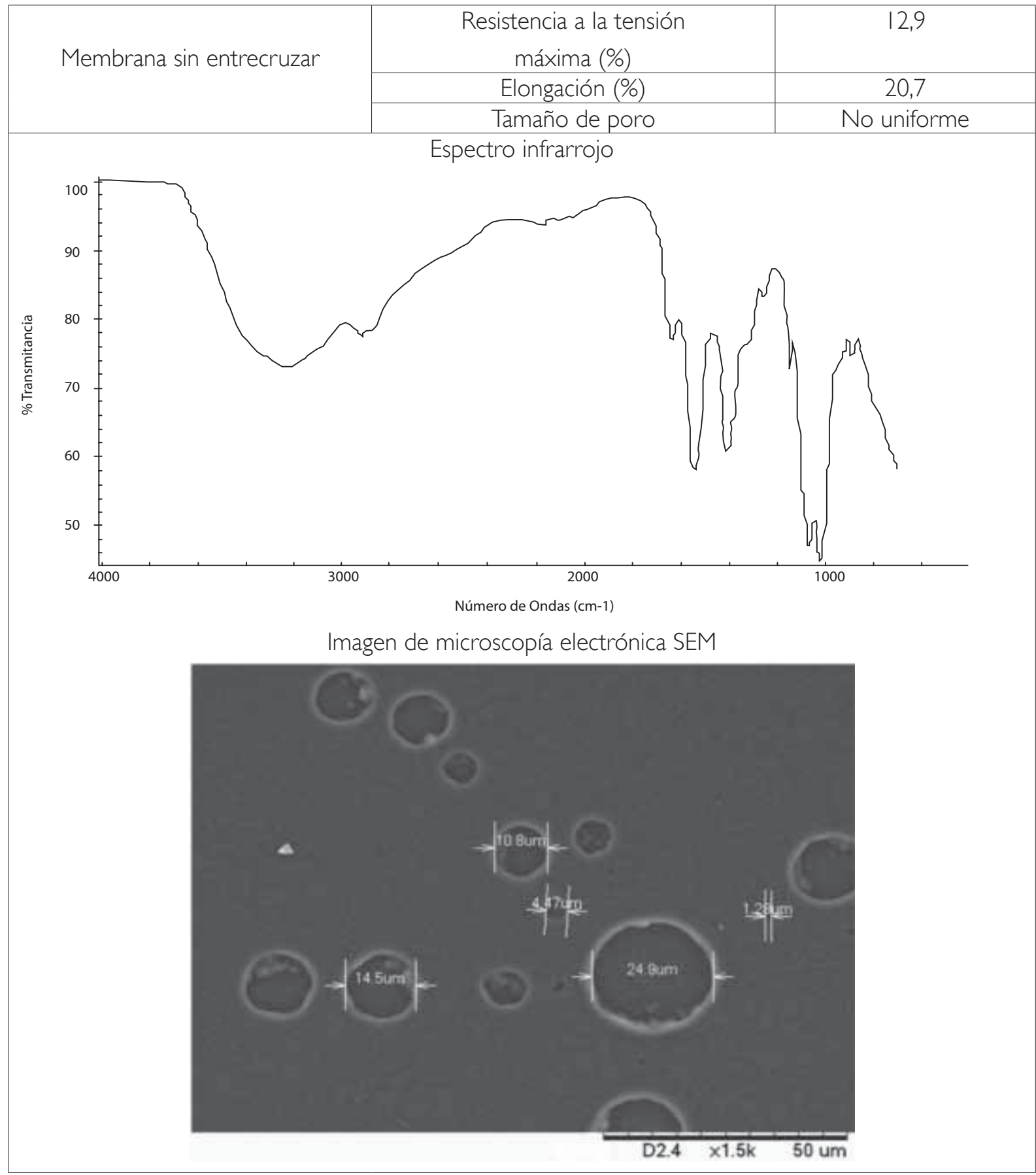

El proceso de adsorción de los metales en las membranas se describió mediante dos modelos: el de Freundlich y el de Langmuir. Se observó, en general, que el comportamiento experimental del fenómeno de adsorción se ajustó con mayor exactitud al modelo de Freundlich, el cual está descrito por la ecuación I.

$$
\log q=\frac{1}{n} \log C_{e}+\log K
$$

Ecuación 1

Donde $\quad$ q: índice de remoción

Ce: concentración de equilibrio

$\mathrm{n}$ : constante de Freundlich

K: constante de Freundlich 
Cuadro 3. Ficha técnica de la membrana de quitosano al $\mathrm{I}, 75 \% \mathrm{~m} / \mathrm{v}$ entrecruzada con glutaraldehído al 0,08\%v/v .

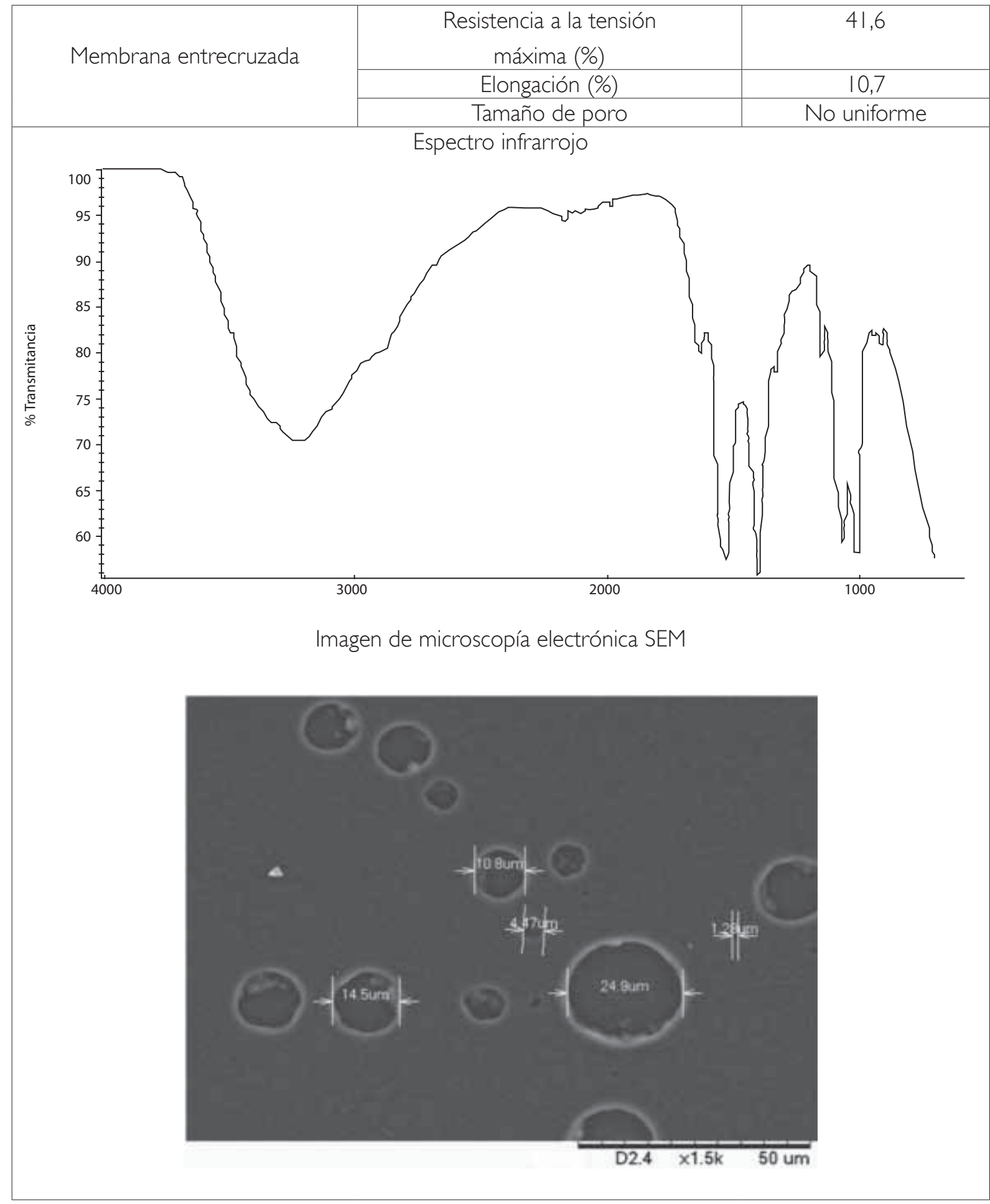

Los datos se analizaron de manera similar, mediante Donde el modelo de Langmuir, descrito por la ecuación 2.

$$
\frac{C_{e}}{q}=\frac{1}{q_{\max }}+\frac{C_{e}}{q_{\max }} \text { Ecuación 2 }
$$

q: $\quad$ índice de remoción

Ce: $\quad$ concentración de equilibrio

b: $\quad$ constante de Langmuir En esta sección se muestran las isotermas de Freundlich obtenidas para los diferentes metales. 


\section{Cromo}

En el caso del cromo, se prepararon disoluciones a partir de dicromato de potasio grado analítico, obteniéndose concentraciones de 4,47; 8,93; I3,40 y 17,87 mg/L de cromo.

\section{Cobre}

En el caso del cobre, se realizó la filtración de disoluciones preparadas a partir de un patrón de cobre para Absorción Atómica de 1000 mg/L. Las concentraciones empleadas fueron: I,0; 4,5; 8,0 y I I,5 mg/L.

\section{Cadmio}

En el caso del cadmio, se realizó la filtración de disoluciones preparadas a partir de un patrón de cadmio para Absorción Atómica de 1000 mg/L. Las concentraciones empleadas fueron: I,0; 4,5; 8,0 y | |,5 mg/L.

El cuadro 7 muestra los resultados obtenidos para el estudio de la adsorción de cromo, cobre y cadmio en las membranas al $1,75 \% \mathrm{~m} / \mathrm{v}$ de quitosano entrecruzadas con glutaraldehído al 0,08\% v/v. Como resultado del estudio de las isotermas de Freundlich, se observaron en general valores de K

$\mathrm{Cr}$, Freundlich, E

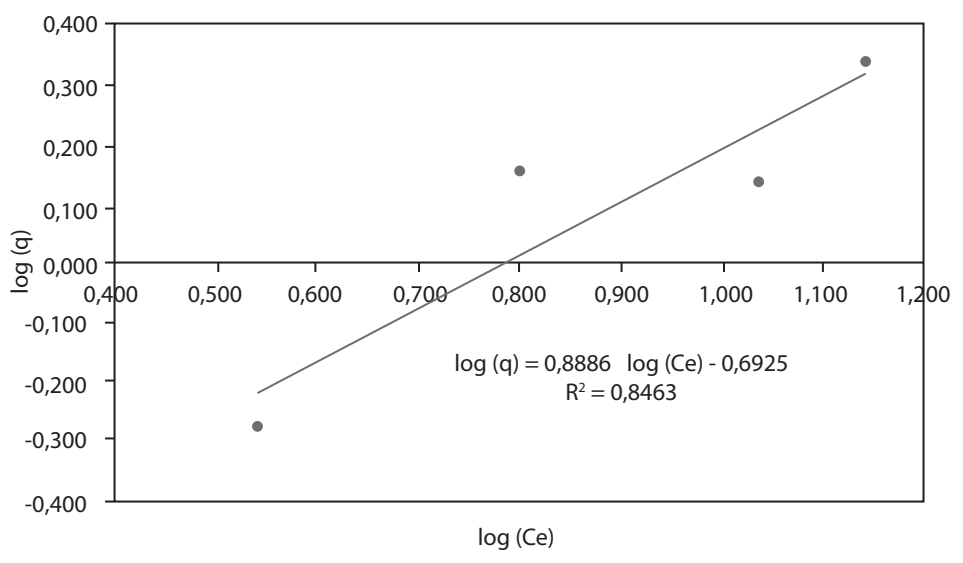

Figura 6. Isoterma de Freundlich obtenida para la filtración de disoluciones de cromo con una membrana de quitosano $1,75 \% \mathrm{~m} / \mathrm{v}$ entrecruzada con glutaraldehído $0,08 \% \mathrm{v} / \mathrm{v}$.

Cuadro 4.Valores experimentales para el cálculo de las isotermas de Freundlich y Langmuir de las disoluciones de cromo.

\begin{tabular}{|c|c|c|c|c|c|c|}
\hline Disolución & $\begin{array}{c}\text { Conc. Inicial } \\
\text { (Co) } \\
(\mathrm{mg} / \mathrm{L})\end{array}$ & $\begin{array}{c}\text { Conc. Equilibrio } \\
\text { (Ce) } \\
(\mathrm{mg} / \mathrm{L})\end{array}$ & $\begin{array}{l}\text { Índice de remoción } \\
\text { (q) } \\
\text { (mg Cr/g } \\
\text { adsorbente) }\end{array}$ & $\log \mathrm{Ce}$ & $\log q$ & $\begin{array}{c}\mathrm{Ce} / \mathrm{q} \\
(\mathrm{mg} / \mathrm{L})\end{array}$ \\
\hline $\mathrm{Crl}$ & 4,47 & 3,49 & 0,535 & 0,543 & $-0,272$ & 6,52 \\
\hline $\mathrm{Cr} 2$ & 4,47 & 3,76 & 0,404 & 0,575 & $-0,393$ & 9,31 \\
\hline $\mathrm{Cr} 3$ & 8,93 & 6,32 & 1,428 & 0,801 & 0,155 & 4,43 \\
\hline $\mathrm{Cr} 4$ & 8,93 & 6,50 & 1,387 & 0,813 & 0,142 & 4,69 \\
\hline $\mathrm{Cr} 5$ & 13,40 & 10,89 & 1,375 & 1,037 & 0,138 & 7,91 \\
\hline $\mathrm{Cr} 6$ & 13,40 & 11,34 & 1,176 & 1,055 & 0,070 & 9,65 \\
\hline $\mathrm{Cr} 7$ & 17,87 & 13,88 & 2,182 & 1,142 & 0,339 & 6,36 \\
\hline $\mathrm{Cr} 8$ & 17,87 & 13,65 & 2,407 & 1,135 & 0,382 & 5,67 \\
\hline
\end{tabular}

Nota: $\mathrm{Crl}$, Cr3, Cr5 y Cr7 fueron filtradas utilizando una membrana entrecruzada. Cr2, Cr4, Cr6 y Cr8 fueron filtradas utilizando una membrana sin entrecruzar. 


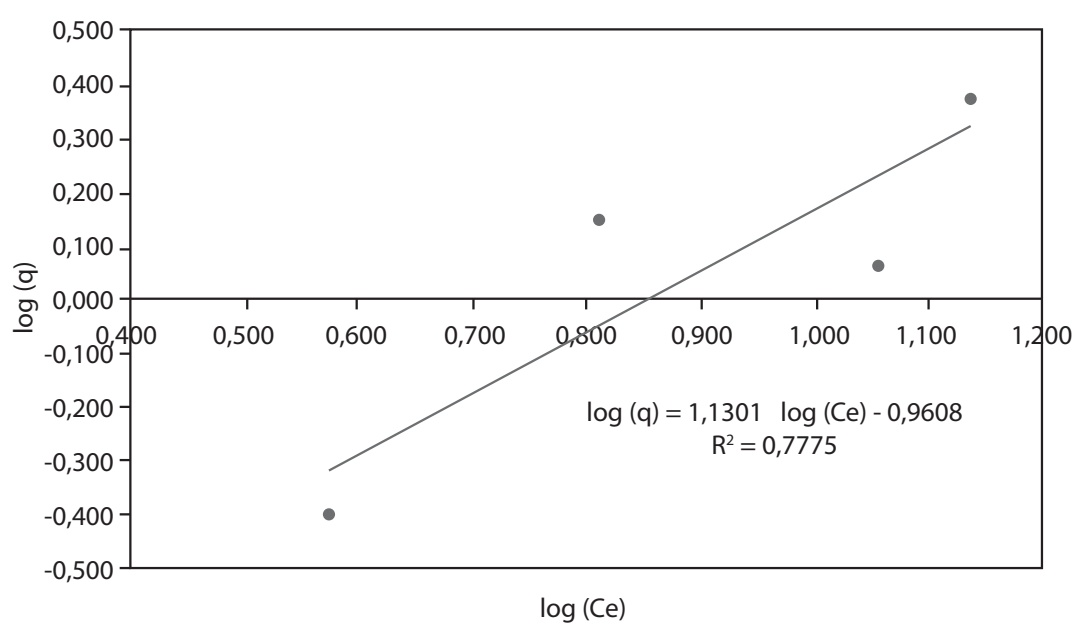

Figura 7. Isoterma de Freundlich obtenida para la filtración de disoluciones de cromo con una membrana de quitosano $1,75 \% \mathrm{~m} / \mathrm{v}$.

$\mathrm{Cr}$, Freundlich, E

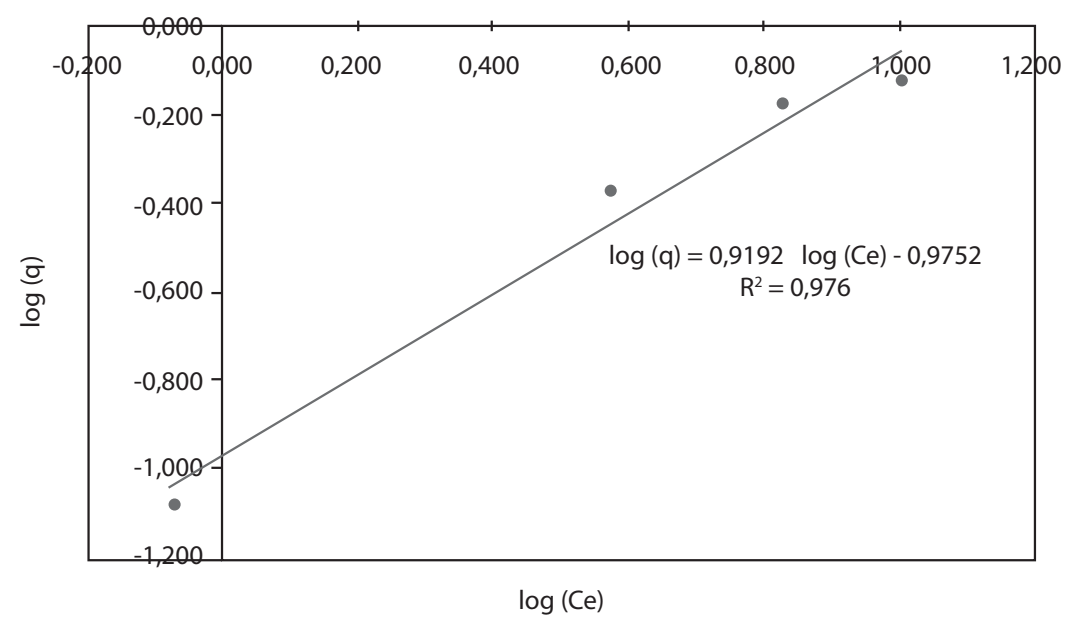

Figura 8. Isoterma de Freundlich obtenida para la filtración de disoluciones de cobre con una membrana de quitosano $1,75 \% \mathrm{~m} / \mathrm{v}$ entrecruzada con glutaraldehído $0,08 \% \mathrm{v} / \mathrm{v}$.

inferiores a 0,4 y valores de $n$ superiores a I, lo cual indica que no se dio una gran interacción entre el quitosano de las membranas y los metales presentes en las disoluciones.

En este punto, es importante mencionar que el tiempo de residencia de los metales en contacto con la membrana fue bajo, dada la naturaleza del equipo y el flujo usado. Esto pudo haber incidido directamente en la eficiencia de la adsorción.
A partir de los resultados experimentales, se observó que el metal que fue removido en mayor medida, con base en los valores de q, fue el cromo $(21,8 \%)$, como se demuestra en el cuadro 8 , seguido del cadmio (17,3\%) y el cobre (13,6\%). 
Cuadro 5. Valores experimentales para el cálculo de las isotermas de Freundlich y Langmuir de las disoluciones de cobre.

\begin{tabular}{|c|c|c|c|c|c|c|}
\hline Disolución & $\begin{array}{l}\text { Conc. Inicial } \\
\text { (Co) } \\
(\mathrm{mg} / \mathrm{L})\end{array}$ & $\begin{array}{c}\text { Conc. Equilibrio } \\
\text { (Ce) } \\
(\mathrm{mg} / \mathrm{L})\end{array}$ & $\begin{array}{c}\text { Índice de remoción } \\
\text { (q) } \\
\text { (mg Cu/g } \\
\text { adsorbente) }\end{array}$ & $\log \mathrm{Ce}$ & $\log q$ & $\begin{array}{c}\mathrm{Ce} / \mathrm{q} \\
(\mathrm{mg} / \mathrm{L})\end{array}$ \\
\hline Cul & 1,0 & 0,85 & 0,08 & $-0,072$ & $-1,077$ & 10,12 \\
\hline $\mathrm{Cu} 2$ & 1,0 & 0,88 & 0,07 & $-0,055$ & $-1,17 \mid$ & 13,08 \\
\hline Cu3 & 4,5 & 3,73 & 0,42 & 0,572 & $-0,376$ & 8,87 \\
\hline Cu4 & 4,5 & 3,80 & 0,40 & 0,580 & $-0,399$ & 9,52 \\
\hline Cu5 & 8,0 & 6,77 & 0,67 & 0,831 & $-0,173$ & 10,10 \\
\hline Cu6 & 8,0 & 7,03 & 0,55 & 0,847 & $-0,257$ & $|2,7|$ \\
\hline Cu7 & 11,5 & 10,13 & 0,75 & 1,006 & $-0,127$ & 13,56 \\
\hline Cu8 & 11,5 & 10,39 & 0,63 & 1,017 & $-0,198$ & $|6,4|$ \\
\hline
\end{tabular}

Nota: Cul, Cu3, Cu5 y Cu7 fueron filtradas utilizando una membrana entrecruzada. Cu2, Cu4, Cu6 y Cu8 fueron filtradas utilizando una membrana sin entrecruzar.

Cr, Freundlich, E

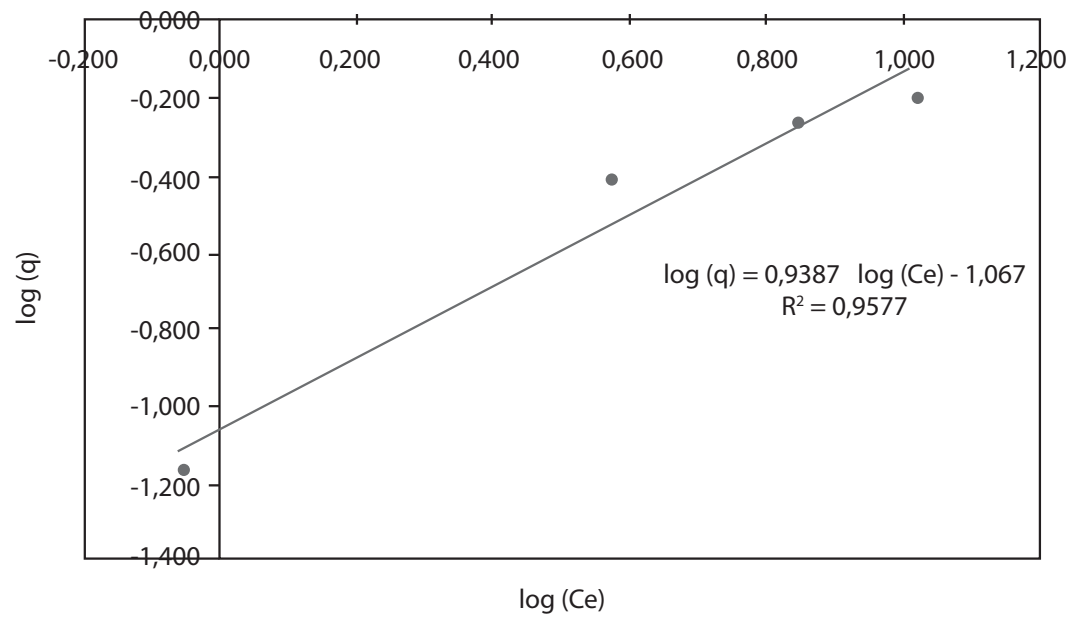

Figura 9. Isoterma de Freundlich obtenida para la filtración de disoluciones de cobre con una membrana de quitosano I,75\% $\mathrm{m} / \mathrm{v}$. 
Cuadro 6.Valores experimentales para el cálculo de las isotermas de Freundlich y Langmuir para las disoluciones de cadmio.

\begin{tabular}{|c|c|c|c|c|c|c|}
\hline Disolución & $\begin{array}{l}\text { Conc. Inicial } \\
\text { (Co) } \\
(\mathrm{mg} / \mathrm{L})\end{array}$ & $\begin{array}{c}\text { Conc. Equilibrio } \\
\text { (Ce) } \\
(\mathrm{mg} / \mathrm{L})\end{array}$ & $\begin{array}{l}\text { Índice de remoción } \\
\text { (q) } \\
\text { (mg Cd/g } \\
\text { adsorbente) }\end{array}$ & $\log \mathrm{Ce}$ & $\log q$ & $\begin{array}{c}\mathrm{Ce} / \mathrm{q} \\
(\mathrm{mg} / \mathrm{L})\end{array}$ \\
\hline $\mathrm{Cul}$ & 1 & 0,68 & 0,18 & $-0,1694$ & $-0,75$ & 3,83 \\
\hline $\mathrm{Cu} 2$ & I & 0,85 & 0,09 & $-0,0721$ & $-1,06$ & 9,69 \\
\hline Cu3 & 4,5 & 3,85 & 0,36 & 0,5855 & $-0,45$ & 10,83 \\
\hline Cu4 & 4,5 & 4,10 & 0,23 & 0,5966 & $-0,64$ & 17,94 \\
\hline Cu5 & 8 & 7,08 & 0,50 & 0,85 & $-0,3$ & 14,05 \\
\hline Cu6 & 8 & 7,39 & 0,35 & 0,8685 & $-0,46$ & 21,12 \\
\hline Cu7 & 11,5 & 10,32 & 0,64 & 1,0139 & $-0,19$ & 16,06 \\
\hline Cu8 & 11,5 & 10,63 & 0,50 & 1,0267 & $-0,31$ & 21,48 \\
\hline
\end{tabular}

Nota: CdI, Cd3, Cd5 y Cd7 fueron filtradas utilizando una membrana entrecruzada. Cd2, Cd4, Cd6 y Cd8 fueron filtradas utilizando una membrana sin entrecruzar.

Cd, Freundlich, E

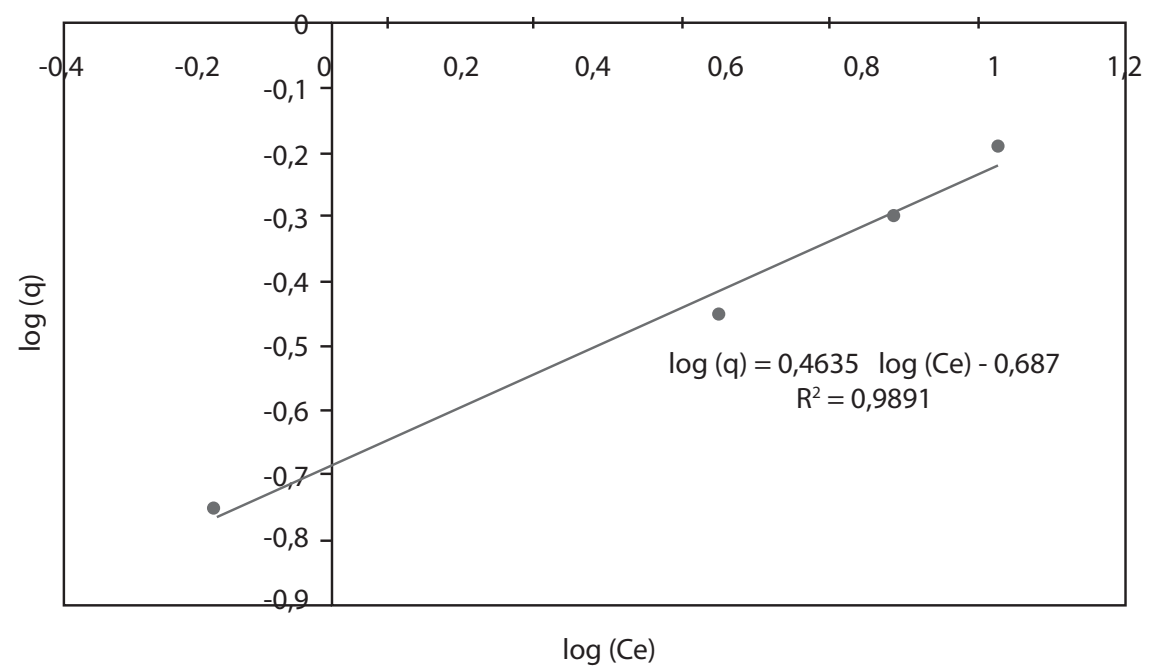

Figura 10. Isoterma de Freundlich obtenida para la filtración de disoluciones de cadmio con una membrana de quitosano $1,75 \% \mathrm{~m} / \mathrm{v}$ entrecruzada con glutaraldehído $0,08 \% \mathrm{v} / \mathrm{v}$.

Cuadro 7. Resultados de adsorción de cromo, cobre y cadmio.

\begin{tabular}{|c|c|c|c|c|}
\hline Metal & Tipo de membrana & $\mathbf{K}$ & $\mathbf{n}$ & $\mathbf{r}^{2}$ \\
\hline Cromo & Entrecruzada & 0,203 & $\mathrm{I}, 125$ & 0,8463 \\
\hline Cromo & Sin entrecruzar & 0,109 & 0,885 & 0,775 \\
\hline Cobre & Entrecruzada & 0,106 & $\mathrm{I}, 088$ & 0,9760 \\
\hline Cobre & Sin entrecruzar & 0,085 & $\mathrm{I}, 065$ & 0,9577 \\
\hline Cadmio & Entrecruzada & 0,206 & 2,157 & $0,989 \mathrm{I}$ \\
\hline Cadmio & Sin entrecruzar & $0,38 \mathrm{I}$ & $\mathrm{I}, 493$ & $0,995 \mathrm{I}$ \\
\hline
\end{tabular}


Cd, Freundlich, SE

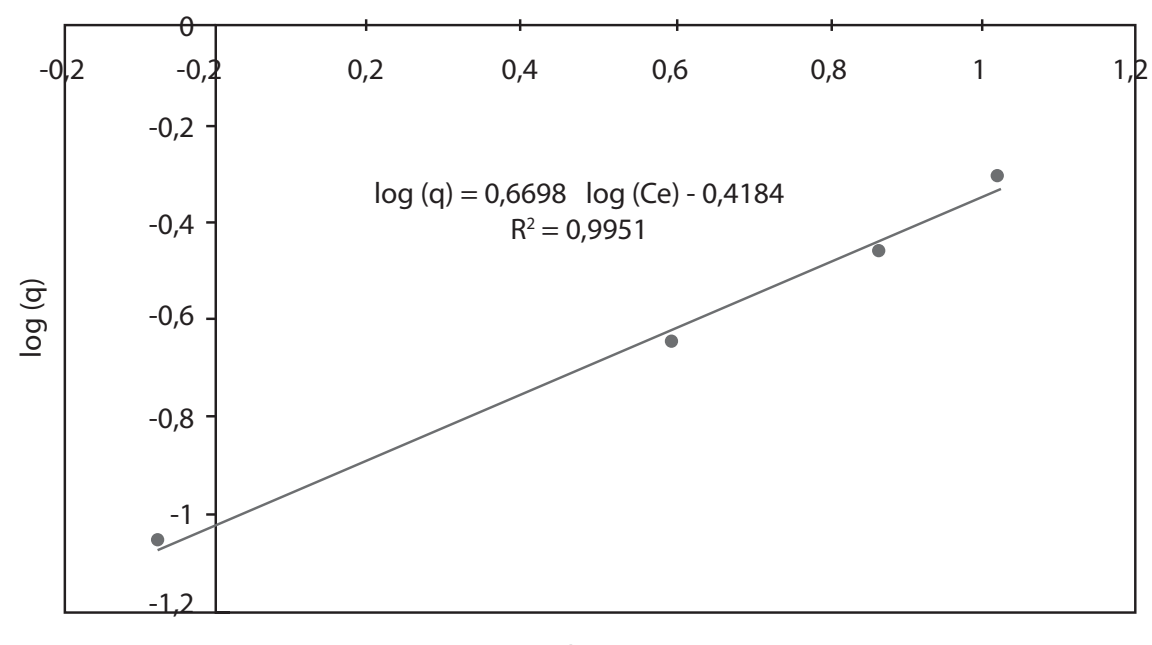

$\log (\mathrm{Ce})$

Figura II. Isoterma de Freundlich obtenida para la filtración de disoluciones de cadmio con una membrana de quitosano $1,75 \% \mathrm{~m} / \mathrm{v}$.

Cuadro 8. Porcentajes de remoción obtenidos para los tres metales en estudio.

\begin{tabular}{|c|c|}
\hline Metal & $\begin{array}{c}\text { Porcentaje de } \\
\text { remoción }\end{array}$ \\
\hline Cromo & $21,8 \%$ \\
\hline Cadmio & $17,3 \%$ \\
\hline Cobre & $13,6 \%$ \\
\hline
\end{tabular}

\section{Conclusiones}

- Se logró preparar dos tipos de membrana con condiciones aptas para la filtración: I,75\% m/v en quitosano y $1,75 \% \mathrm{~m} / \mathrm{v}$ entrecruzada con glutaraldehído $0,08 \% \mathrm{v} / \mathrm{v}$.

- Se elaboró una ficha técnica para cada membrana utilizada en la filtración, incluyendo información de espectroscopía infrarroja, microscopía electrónica SEM y propiedades mecánicas.

- No se encontró correlación entre el tamaño de poro de las membranas con respecto al grado de entrecruzamiento.

- Las membranas entrecruzadas mostraron mejores propiedades mecánicas que las no entrecruzadas, como lo demuestran las mediciones de resistencia a la tensión y capacidad de elongación.

- De los tres metales analizados, el cromo fue el que se removió en mayor medida, mediante la filtración con membranas entrecruzadas.

- De los dos modelos de isotermas de adsorción analizados, el de Freundlich se ajustó mejor que el de Langmuir a los resultados obtenidos.

\section{Recomendaciones}

- Es necesario perfeccionar el método de preparación de membranas en aspectos tales como: concentraciones de quitosano y tipo de entrecruzante empleado. 
- El equipo de filtración debe ser mejorado, para poder tener mejor control sobre las variables: presión y flujo de agua.

- Para una futura propuesta, podría realizarse el experimento de adsorción en un sistema de bache, donde se agrega el adsorbente de quitosano al agua contaminada en un recipiente agitado, sin flujo de agua.

- Analizar otros tipos de metales y su interacción con membranas de quitosano.

\section{Agradecimientos}

Los autores expresan su agradecimiento al Tecnológico de Costa Rica (TEC) y en especial a la Vicerrectoría de Investigación y Extensión por el apoyo tanto financiero como administrativo. También agradecemos al Centro de Investigación en Protección Ambiental (CIPA) y al Laboratorio de Servicios Químicos y Microbiológicos (CEQIATEC) por su respaldo al proyecto. Finalmente, al Instituto Nacional de Aprendizaje (INA), especialmente al Lic. Roy Zamora.

\section{Bibliografía}

Aroua, MK ZF \& Sulaiman, NM. (agosto 2007). Removal of chromium ions from aqueous solutions by polymerenhanced ultrafiltration. Journal of hazardous materials I 47( 3):752-758.

Cañizares, R. (2000). Biosorción de metales pesados mediante el uso de biomasa microbiana. Revista Latinoamericana de Microbiología 42: | 3 | - 143.

Mallevialle, J. OP \& Wierner M. (1998). Tratamiento del agua por procesos de membrana. I ed. España: Mc Graw Hill.
Paulino, AT; Reis, AV; Tambourgi, EB; Nozaki, J \& Muniz, EC. (agosto 2007). Capacity of adsorption of Pb2+ and $\mathrm{Ni} 2+$ from aqueous solutions by chitosan produced from silkworm chrysalides in different degrees of deacetylation. Journal of hazardous materials I47(1-2): I39-147.

Rayment, G. (2005). Cadmium in Sugar Cane and Vegetable Systems of Northeast Australia. Communications in Soil Science and Plant Analysis 36:597-608. Toory, B.M. \& Kee Kwong K.F. (2003). Leaching and Uptake of Heavy Metals From Soils amended with sewage sludge under rainfed sugar cane in Mauritius. Food and Agricultural Research Council.

Renata, M. GB, Faruk, N., Haidi, F. \& Laranjeira, M. (2006). Preparation of chitosan membranes for filtration and concentration of compounds under high pressure process. Polymer Bulletin 56:447-454.

Robinson, L. (2008). Water Resources Research Progress. NOVA Sciences Publishers Inc. p. 156.

Schmuhl, R. KH. \& Keizer, K. (enero 200I). Adsorption of $\mathrm{Cu}(\mathrm{II})$ and $\mathrm{Cr}(\mathrm{VI})$ ions by chitosan: Kinetics and equilibrium studies. Water SA 27(I).

Song S. Y.B.; Shim W.; Hudson S. \& Hwang, T. (noviembre 2007). Synthesis of Biocompatible CS-g-CMS ion exchangers and their adsorption Behavior for heavy metal ions. Journal of industrial and engineering chemistry 13(6): 1009-1016.

Trimukhe, K.D. \& Varma, A.J. (enero 2008). Complexation of heavy metals by crosslinked chitin and its deacetylated derivatives. Carbohydrates Polymers 7I ( I):66-73.

Varios. (2000). Standard methods for the examination of water and wastewater. Vol. 20.

Velásquez C.L. (2006). Quitina y quitosano: materiales del pasado para el presente y el futuro. Avances en Química I (2): |5-2 I. 